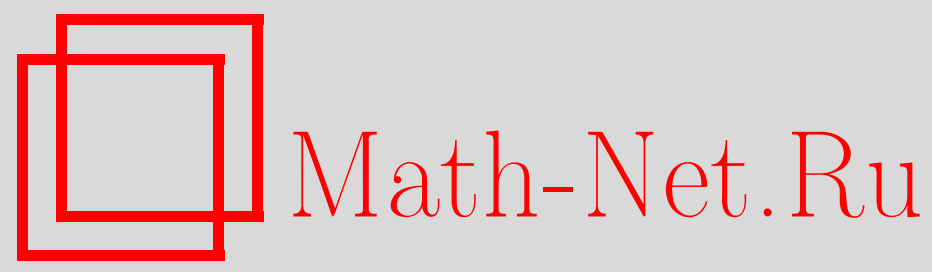

А. Н. Зубков, Об одном матричном представлении свободной группы, Матем. заметки, 1998, том 64, выпуск 6, 863870

DOI: https://doi.org/10.4213/mzm1465

Использование Общероссийского математического портала Math-Net.Ru подразумевает, что вы прочитали и согласны с пользовательским соглашением http://www. mathnet.ru/rus/agreement

Параметры загрузки:

IP: 34.239 .49 .27

26 апреля 2023 г., 13:04:40

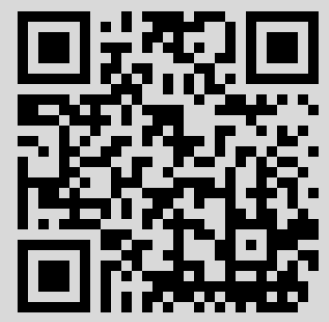




\title{
ОБ ОДНОМ МАТРИЧНОМ ПРЕДСТАВЛЕНИИ СВОБОДНОЙ ГРУППЫ
}

\author{
А. Н. Зубков
}

Исследуется аналог известного представления Санова свободной неабелевой группы матрицами размера $\geqslant 3$. Вместо трансвекций, как в представлении Санова, берутся матрицы с "заполненным" первым (вторым и т.д.) столбцом, кроме пересечения с диагональю. На диагонали стоят единицы, а на остальных местах - нули. На "заполняемые" места ставится один и тот же параметр $k$. Доказано, что при $|k| \geqslant 5$ эти матрицы порождают свободную группу. Однако, при $k=2$ это уже не так.

Библиограффия: 4 названия.

Эта заметка была написана автором в 1981 году, но нигде не опубликована, если не считать краткого сообщения [1]. Однако, найденное доказательство точности представления свободной группы, обобщающее известное представление Санова [2], на наш взгляд заслуживает внимания специалистов по теории линейных груп. В свое время различные усиления результата Санова были достаточно популярной темой, о чем свидетельствует обзор [3]. Однако, насколько это известно автору, не было никаких попыток обобшить представление Санова на случай размерности $\geqslant 3$. Исследуемое в этой работе представление было предложено В.Н. Ремесленниковым (устное сообщение).

Напомним некоторые определения. Представлением Санова обычно назьвают гомоморфизм свободной групшы $F(x, y)$, со свободньми порождающими $x, y, \mathrm{SL}_{2}(\mathbb{C})$, где $\mathbb{C}$ - поле комплексных чисел, по правилу

$$
x \mapsto\left(\begin{array}{cc}
1 & z \\
0 & 1
\end{array}\right), \quad y \mapsto\left(\begin{array}{cc}
1 & 0 \\
z & 1
\end{array}\right) .
$$

Хорошо известно, что при $|z| \geqslant 2$ это представление точно [2]. Там же можно найти метод "восстановления" слова $v(x, y) \in F(x, y)$ по его образу в $\mathrm{SL}_{2}(\mathbb{C})$ в частном случае $z=2$.

Здесь мы покажем, что матрицы

$$
U_{1 n}(k)=\left(\begin{array}{cccc}
1 & 0 & \ldots & 0 \\
k & 1 & \ldots & 0 \\
\vdots & \vdots & \ddots & \vdots \\
k & 0 & \ldots & 1
\end{array}\right), \ldots, \quad U_{n n}(k)=\left(\begin{array}{cccc}
1 & 0 & \ldots & k \\
0 & 1 & \ldots & k \\
\vdots & \vdots & \ddots & \vdots \\
0 & 0 & \ldots & 1
\end{array}\right)
$$

при определенном ограничении на вешественный параметр $k$ порождают свободную группу ранга $n$. Сушественно, что параметр $k$ предполагается вешественньм, поскольку 
при доказательстве используется постоянство знака некоторых координат "пробного" вектора.

Прежде чем мы сформулируем основной результат, введем для удобства некоторые обозначения. Пусть $e_{j k}$ - стандартная матрица с единицей в $j$-й строке и $k$-м столбце и с нулями на остальных местах. Как обычно, $E_{n}=\sum_{1 \leqslant i \leqslant n} e_{i i}$ - единичная матрица. Пусть $s_{i n}=\sum_{1 \leqslant j \leqslant n} e_{j i}, 1 \leqslant i \leqslant n$.

Имеет место

Tеорема 1. Группа $G_{n}(k)$, порождденная матрицами $U_{i n}(k)=E_{n}+k s_{i n}, 1 \leqslant i \leqslant n$, свободна при всех $n \geqslant 3$ u $|k| \geqslant 5$. Группа $G_{n}(2)$ не свободна при всех $n \geqslant 3$. Кроме того, группа $G_{3}(2)$ содержит главную конгруэни-подгруппу по модулю 32.

Отметим некоторые очевидные свойства матриц $U_{i n}$. Для любого $s \in \mathbb{Z} U_{i n}(k)^{s}=$ $U_{i n}(s k)$. Далее, для любого вектора $a=\left(a_{1}, \ldots, a_{n}\right) \in \mathbb{R}^{n}$ мы имеем $U_{i n}(l) a^{t}=b^{t}$, где $b=\left(b_{1}, \ldots, b_{n}\right)$ и

$$
b_{j}= \begin{cases}a_{i} & \text { при } j=i, \\ a_{j}+l a_{i} & \text { при } j \neq i ;\end{cases}
$$

здесь $t$ означает транспонирование.

\section{1. Группа $G_{n}(2), n \geqslant 3$.}

Лемма 1. Пусть $2 \leqslant r \leqslant n$. Тогда соответствие $U_{i r}(k) \leftrightarrow U_{i n}(k), 1 \leqslant i \leqslant r$, продолжсается до вложсения $G_{r}(k)$ в $G_{n}(k)$.

ДокАЗАТЕЛЬСТво. В кольце матриц $M_{n}(\mathbb{R})$ породим подкольцо $K$ при помощи элементов $s_{1 n}, \ldots, s_{r n}$. Ясно, что $K$ является гомоморфным образом абсолютно свободного ассоциативного кольца $Z\left\langle x_{1}, \ldots, x_{r}\right\rangle$ относительно гомоморфизма $x_{i} \mapsto s_{i n}$,

Обозначим ядро этого гомоморфизма через $I$. Несложные выгисления показывают, что $x_{i}^{2}, x_{i} x_{j} x_{i}-x_{i}, x_{i} x_{j} x_{l}-x_{i} x_{l}$ лежат в $I$ для всех $i \neq j, i \neq l, j \neq l$. Более того, идеал $I$ порождается этими элементами.

Действительно, по модулю этих соотношений любой элемент кольца $Z\left\langle x_{1}, \ldots, x_{r}\right\rangle$ преобразуется в выражение вида

$$
u \cdot 1+\sum_{\substack{1 \leqslant i, j \leqslant r \\ i \neq j}} v_{i j} x_{i} x_{j} .
$$

Если оно лежит в идеале $I$, то, специализируя его в $M_{n}(\mathbb{R})$, получим равенство

$$
0=u \cdot E_{n}+\sum_{\substack{1 \leqslant i, j \leqslant r \\ i \neq j}} v_{i j} s_{i n} s_{j n} .
$$

После несложного пересчета имеем

$$
0=u \cdot E_{n}+\sum_{i \neq j} v_{i j}\left(\sum_{k \neq i} e_{k i}\right) .
$$

Преобразуя сумму еще раз, получим выражение

$$
u \cdot E_{n}+\sum_{\substack{1 \leqslant k \leqslant n \\ 1 \leqslant j \leqslant r}} e_{k j}\left(\sum_{\substack{i \neq k, j \\ 1 \leqslant i \leqslant r}} v_{i j}\right)=0 .
$$


Случай $r=n=2$ разбирается непосредственно: $u=v_{12}=v_{21}=0$. Пусть $n \geqslant 3$ и $r=2$. Тогда при $e_{31}$ будет коэффициент $v_{21}$, а при $e_{32}-$ коэффициент $v_{12}$. Следовательно, $v_{12}=v_{21}=0$ и, тем более, $u=0$.

Осталось рассмотреть случай $n \geqslant 3, r \geqslant 3$. Пусть $1 \leqslant j, k_{1}, k_{2} \leqslant r$, где $j \neq k_{1}$, $j \neq k_{2}, k_{1} \neq k_{2}$. Тогда

$$
\sum_{i \neq j, k_{1}} v_{i j}=\sum_{i \neq j, k_{2}} v_{i j}=0 .
$$

В частности, $v_{k_{1} j}=v_{k_{2} j}$. В силу произвольности выбора $j, k_{1}, k_{2}$ все $v_{i j}$ равны между собой и, следовательно, равны 0. Отсюда $u=0$.

Размер матриц $n$ никоим образом не влиял на наши рассуждения. Следовательно, $K \cong Z\left\langle x_{1}, \ldots, x_{r}\right\rangle / I \cong Z\left[s_{1 r}, \ldots, s_{r r}\right]$. В частности, соответствие $s_{i n} \leftrightarrow s_{i r}, 1 \leqslant i \leqslant r$, продолжается до изоморфизма $Z\left[s_{1 r}, \ldots, s_{r r}\right]$ на $Z\left[s_{1 n}, \ldots, s_{r n}\right]$. Очевидно, при этом $U_{i r}$ переходит в $U_{i n}$, что и требуется. Лемма доказана.

ЛЕмма 2. Произвольная перестановка порождающих $U_{i n}(k)$ продолжается до автоморфизма группь $G_{n}(k)$.

ДоКАЗАТЕЛЬСТВО. Как обычно, лемму достаточно доказать для транспозиций. Пусть $U_{i n}(k) \mapsto U_{j n}(k), U_{j n}(k) \mapsto U_{i n}(k)$ и $U_{l n}(k) \mapsto U_{l n}(k)$ для $l \neq i, j$. Как мы показали выше, кольцо $Z\left[s_{1 n}, \ldots, s_{n n}\right]$ изоморфно факторкольцу $Z\left\langle x_{1}, \ldots, x_{r}\right\rangle / I$. Несложно продемонстрировать, что подстановка $x_{i} \mapsto x_{j}, x_{j} \mapsto x_{i}, x_{l} \mapsto x_{l}, l \neq i, j$, лишь переставляет порождающие идеала $I$. В частности, она индуцирует автоморфизм $Z\left\langle x_{1}, \ldots, x_{r}\right\rangle / I$, т.е. автоморфизм кольца $Z\left[s_{1 n}, \ldots, s_{n n}\right]$. Очевидно, этот автоморфизм индуцирует искомый автоморфизм группы $G_{n}(k)$. Лемма доказана.

TeOpema 2. Группа $G_{3}(2)$ не свободна.

ДокАЗАТЕЛЬСтво. Обозначим через $T$ матрицу

$$
\left(\begin{array}{ccc}
1 & -1 & -1 \\
0 & 1 & 0 \\
0 & 0 & 1
\end{array}\right)
$$

Нетрудно вычислить, что

$$
Z=T U_{33}(2) T^{-1}=\left(\begin{array}{ccc}
1 & 0 & 0 \\
0 & 1 & 2 \\
0 & 0 & 1
\end{array}\right), \quad Y=T U_{23}(2) T^{-1}=\left(\begin{array}{lll}
1 & 0 & 0 \\
0 & 1 & 0 \\
0 & 2 & 1
\end{array}\right)
$$

В силу результата Санова [2] группа $G_{2}(2)$ состоит из тех и только тех матрищ

$$
\left(\begin{array}{ll}
\alpha & \beta \\
\gamma & \delta
\end{array}\right) \in \mathrm{SL}_{2}(Z)
$$

для которых $\alpha \equiv \delta \equiv 1(\bmod 4), \beta \equiv \gamma \equiv 0(\bmod 2)$.

Отсюда ясно, что группа $\langle Z, Y\rangle$ состоит из всех матриц вида

$$
\left(\begin{array}{lll}
1 & 0 & 0 \\
0 & \alpha & \beta \\
0 & \gamma & \delta
\end{array}\right), \quad \text { где } \quad\left(\begin{array}{cc}
\alpha & \beta \\
\gamma & \delta
\end{array}\right) \in G_{2}(2)
$$


Не представляет большого труда и проверка следуюшего равенства:

$$
X^{k}=\left(\begin{array}{ccc}
1-4 k & -4 k & -4 k \\
2 k & 1+2 k & 2 k \\
2 k & 2 k & 1+2 k
\end{array}\right)
$$

где

$$
X=T U_{13}(2) T^{-1}=\left(\begin{array}{ccc}
-3 & -4 & -4 \\
2 & 3 & 2 \\
2 & 2 & 3
\end{array}\right)=\left(\begin{array}{ccc}
1-4 & -4 & -4 \\
2 & 1+2 & 2 \\
2 & 2 & 1+2
\end{array}\right) .
$$

В дальнейшем нам понадобится еще одна формула, которая также получается прямым вычислением. Для любого

$$
v(Y, Z)=\left(\begin{array}{lll}
1 & 0 & 0 \\
0 & \alpha & \beta \\
0 & \gamma & \delta
\end{array}\right)
$$

и любого $k \in \mathbb{Z}$ имеем

$$
X^{-k} v(Y, Z) X^{k}=\left(\begin{array}{ccc}
1+x & 4 k f+x & 4 k t+x \\
p+\left(4 k^{2}-p\right) r-4 k^{2} z & \alpha+2 k g-x / 2 & \beta+2 k h-x / 2 \\
p+\left(4 k^{2}-p\right) z-4 k^{2} r & \gamma-2 k h-x / 2 & \delta+2 k y-x / 2
\end{array}\right),
$$

где

$$
f=\alpha+\gamma-1, \quad t=\beta+\delta-1, \quad r=\alpha+\beta, \quad z=\gamma+\delta, \quad y=\gamma-\delta,
$$

$g=t-z+1, \quad h=f-z+1, \quad x=8 k^{2}(s-2), \quad p=-2 k(1-4 k), \quad s=\alpha+\beta+\gamma+\delta$.

Положим $\alpha=1+4 m, \delta=1-4 m, \beta=-4 m, \gamma=4 m$. В этом случае $\alpha+\beta=\gamma+\delta=1$. Применяя процедуру Санова, несложно вычислить, что $v(Y, Z)=\left(Z Y^{-1}\right)^{2 m}$. Подставляя это равенство в формулу (1), имеем

$$
X^{-k}\left(Z Y^{-1}\right)^{2 m} X^{k}=\left(\begin{array}{ccc}
1 & 32 k m & -32 k m \\
0 & 1+4 m(1-4 k) & -4 m(1-4 k) \\
0 & 4 m(1-4 k) & 1-4 m(1-4 k)
\end{array}\right) .
$$

Следовательно,

$$
\left(Z Y^{-1}\right)^{-2 m(1-4 k)} X^{-k}\left(Z Y^{-1}\right)^{2 m} X^{k}=\left(\begin{array}{ccc}
1 & 32 k m & -32 k m \\
0 & 1 & 0 \\
0 & 0 & 1
\end{array}\right) .
$$

Совершенно очевидно, что матрица вида

$$
\left(\begin{array}{lll}
1 & 0 & 0 \\
0 & \alpha & \beta \\
0 & \gamma & \delta
\end{array}\right)
$$

коммутирует с любой матрицей типа

$$
\left(\begin{array}{lll}
1 & \pi & \chi \\
0 & 1 & 0 \\
0 & 0 & 1
\end{array}\right)
$$

В частности, мы имеем нетривиальное (т.е. с точностью до сопряжения матрищей $T$ между порождаюшими групшы $\left.G_{3}(2)\right)$ соотношение между $X, Y, Z$ :

$$
\left[\left(Z Y^{-1}\right)^{-2 m(1-4 k)} X^{-k}\left(Z Y^{-1}\right)^{2 m} X^{k},\left(Z Y^{-1}\right)^{2 n}\right]=1,
$$

где $[\cdot, \cdot \cdot]$ - обычньй коммутатор и $k, m, n \in \mathbb{Z}$. Теорема доказана. 
СледСТвиЕ. Группа $G_{n}(2)$ не свободна для любых $n \geqslant 3$.

ДокАЗАТЕльСтво. Действительно, как мы показали вьше, группа $G_{3}(2)$ вкладывается в $G_{n}(2)$.

Следующая теорема дает дополнительную информацию о группе $G_{3}(2)$. Главную конгруэнц-подгрупу в $\mathrm{SL}_{n}(Z)$ по модулю $m$ будем обозначать через $\Gamma_{n}(m)$.

Teopema $3 . G_{3}(2) \geqslant \Gamma_{3}(32)$.

ДокАЗАтЕльство. Полагая в формуле (1) $\alpha=1+4 m, \delta=1-4 m, \beta=4 m$, $\gamma=-4 m$, получим матрицу вида

$$
\left(\begin{array}{ccc}
1 & 0 & 0 \\
16 k m & * & * \\
-16 k m & * & *
\end{array}\right)
$$

где "угол"

$$
\left(\begin{array}{lll}
1 & 0 & 0 \\
0 & * & * \\
0 & * & *
\end{array}\right)
$$

лежит в группе $\langle Y, Z\rangle$. Сокрашая на подходяший элемент из этой групшы, мы получим, в частности, матрицу

$$
M=\left(\begin{array}{ccc}
1 & 0 & 0 \\
16 & 1 & 0 \\
-16 & 0 & 1
\end{array}\right)
$$

Заметим, что подгруппа, порожденная $U_{23}(2), U_{33}(2)$, состоит из всех матриц вида

$$
\left(\begin{array}{ccc}
1 & \alpha+\gamma-1 & \beta+\delta-1 \\
0 & \alpha & \beta \\
0 & \gamma & \delta
\end{array}\right), \quad \text { где }\left(\begin{array}{ll}
\alpha & \beta \\
\gamma & \delta
\end{array}\right) \in G_{2}(2) .
$$

Отсюда, в частности, мы видим, что

$$
L=\left(\begin{array}{ccc}
1 & 0 & 0 \\
0 & 1-16 & -16 \\
0 & 16 & 1+16
\end{array}\right) \in G_{3}(2)
$$

С другой стороны,

$$
S=T^{-1} M T=L=\left(\begin{array}{ccc}
1 & 0 & 0 \\
16 & 1-16 & -16 \\
-16 & 16 & 1+16
\end{array}\right) \in G_{3}(2)
$$

и, в частности,

$$
S L^{-1}=\left(\begin{array}{ccc}
1 & 0 & 0 \\
16 & 1 & 0 \\
-16 & 0 & 1
\end{array}\right) \in G_{3}(2)
$$


Домножая на $U_{13}(2)^{8}$, мы получим трансвекщию

$$
t_{21}(32)=\left(\begin{array}{ccc}
1 & 0 & 0 \\
32 & 1 & 0 \\
0 & 0 & 1
\end{array}\right) \in G_{3}(2)
$$

Аналогично,

$$
L S^{-1} U_{13}^{8}=t_{31}(32)=\left(\begin{array}{ccc}
1 & 0 & 0 \\
0 & 1 & 0 \\
32 & 0 & 1
\end{array}\right) \in G_{3}(2) .
$$

Пусть $\sigma \in S_{n}$-произвольная подстановка на $n$ символах. Как мы уже знаем, $U_{i n}(k) \mapsto$ $U_{\sigma(i), n}(k)$ - это автоморфизм групшы $G_{n}(k)$. Более того, индукцией по длине элемента $v \in G_{n}(k)$ несложно показать, что $v^{\sigma} \cdot a^{t}=\left(v \cdot\left(a^{\sigma}\right)^{t}\right)^{\sigma}$, где $a \in \mathbb{R}^{n}$ и $\left(a^{\sigma}\right)_{i}=a_{\sigma^{-1}(i)}$, $1 \leqslant i \leqslant n$.

Рассмотрим транспозицию $\tau: U_{13}(2) \mapsto U_{23}(2), U_{23}(2) \mapsto U_{13}(2)$. Фиксируем стандартньй базис пространства $\mathbb{R}^{3}: e_{1}=(1,0,0), e_{2}=(0,1,0), e_{3}=(0,0,1)$. Тогда, например,

$$
t_{21}(32)^{\tau} e_{1}^{t}=\left(t_{21}(32) e_{2}^{t}\right)^{\tau}=\left(e_{2}^{\tau}\right)^{t}=e_{1}^{t}
$$

Аналогично,

$$
t_{21}(32)^{\tau} e_{2}^{t}=(32,1,0)^{t}, \quad t_{21}(32)^{\tau} e_{3}^{t}=e_{3}^{t} .
$$

Таким образом, $t_{21}(32)^{\tau}=t_{12}(32) \in G_{3}(2)$. Тем же способом мы можем "выташить" и все остальные трансвекции $t_{i j}(32), 1 \leqslant i \neq j \leqslant 3$. Хорошо известно, что $\Gamma_{n}(m)(n \geqslant 3)$ порождается трансвекциями $t_{i j}(m), 1 \leqslant i \neq j \leqslant n$ (см. [4, §12, леммы 6, 7]). Теорема доказана.

\section{2. Точность представления.}

Теорема 4. Группа $G_{n}(k)$ свободна, со свободными порождающими $U_{i n}(k), 1 \leqslant$ $i \leqslant n$, при всех $n \geqslant 3 u|k| \geqslant 5$.

ДоКАЗАТЕЛЬСТВо. Без потери обшности можно считать, что $k>0$. Пусть $v=v_{s}=$ $U_{r_{s} n}(k)^{l_{s}} \cdots U_{r_{1} n}(k)^{l_{1}}$ - произвольное редуцированное слово от порождающих $U_{i n}(k)$, т.е. $r_{i} \neq r_{i+1}, 1 \leqslant i \leqslant s-1$ и все $l_{1}, \ldots, l_{s} \in \mathbb{Z} \backslash\{0\}$.

Как обычно, через $e_{1}, \ldots, e_{n}$ обозначим стандартньци базис пространства $\mathbb{R}^{n}$.

Наша цель - показать, что $v \cdot e_{r_{1}}^{t}=b^{t}$, причем у вектора $b$ все координаты ненулевые. Более того, все они, кроме быть может $b_{r_{s}}$, одного знака. В частности, $v \cdot e_{r_{1}}^{t} \neq e_{r_{1}}^{t}$ и, следовательно, $v \neq E_{n}$.

Будем говорить, что вектор $b$ удовлетворяет условию $(l)$ с параметрами $a, d>0$, если все его координаты ненулевые и все $b_{i}$, где $i \neq l$, одного знака, причем $0<a \leqslant\left|b_{i} / b_{l}\right|$. Кроме того, для любых $i, j \neq l$ и $i \neq j$ либо $1 \leqslant\left|b_{i} / b_{j}\right| \leqslant d$, либо $1 / d \leqslant\left|b_{i} / b_{j}\right| \leqslant 1$.

Индукцией по длине $v$ мы покажем, что $v \cdot e_{r_{1}}^{t}$ удовлетворяет условию $\left(r_{s}\right)$ с параметрами $a, d$, зависяшими только от $k$. При этом по ходу доказательства будут возникать некоторые неравенства для $a, d$, необходимые для индуктивного перехода. Итак, от нас требуется доказать, что система этих неравенств совместна при любом $k \geqslant 5$.

База индукции. Положим $1 / k<a<k, 1 \leqslant d<k$. Тогда вектор

$$
U_{r_{1} n}(k)^{l_{1}} \cdot e_{r_{1}}^{t}=(k l_{1}, \ldots, \underbrace{1, \ldots, 1}_{r_{1}}, \ldots, k l_{1})^{t}
$$


очевидно удовлетворяет условию $\left(r_{1}\right)$ с параметрами $a, d$.

Индуктивный переход. Упрощая обозначения, положим $i=r_{s}, j=r_{s+1}, i \neq j$. Пусть вектор $v_{s} \cdot e_{r_{1}}^{t}=b^{t}$ удовлетворяет условию $(i)$ с параметрами $a, d$.

Пусть $U_{j n}(k)^{l} \cdot b^{t}=\left(b^{\prime}\right)^{t}=\left(b_{1}^{\prime}, \ldots, b_{n}^{\prime}\right)^{t}$. Здесь $b_{j}=b_{j}^{\prime}$ и $b_{m}^{\prime}=b_{m}+k l b_{j}$ для всех $m \neq j$. По условию $b_{j}^{\prime}=b_{j} \neq 0$. Следовательно, $\left|b_{m}^{\prime} / b_{j}^{\prime}\right|=\left|b_{m} / b_{j}+l k\right| \geqslant k-\left|b_{m} / b_{j}\right| \geqslant a$ для всех $m \neq j$. Если $m=i$, то $\left|b_{i} / b_{j}\right| \leqslant 1 / a$ и для индуктивного перехода нужно потребовать $k-1 / a \geqslant a$. Если $m \neq i$, то $\left|b_{m} / b_{j}\right| \leqslant d$, откуда $k-d \geqslant a$. Первое неравенство эквивалентно

$$
\frac{k-\sqrt{k^{2}-4}}{2} \leqslant a \leqslant \frac{k+\sqrt{k^{2}-4}}{2} .
$$

Учитьвая

$$
k-d \leqslant k-1<\frac{k+\sqrt{k^{2}-4}}{2}
$$

в итоге имеем два неравенства

$$
\frac{k-\sqrt{k^{2}-4}}{2} \leqslant a \leqslant k-d \quad \text { и } \quad 1 \leqslant d<k .
$$

Пусть $m \neq s, m \neq j, s \neq j$. Обозначим через $x$ модуль

$$
\left|\frac{b_{m}^{\prime}}{b_{s}^{\prime}}\right|=\left|\frac{k l+b_{m} / b_{j}}{k l+b_{s} / b_{j}}\right| \text {. }
$$

Дальнейшие рассуждения зависят от знака $l$.

a) Пусть $l>0$. Если $m, s \neq i$, то по условию коэффициенты $b_{j}, b_{m}, b_{s}$ одного знака, т.е. $b_{m} / b_{j}>0, b_{s} / b_{j}>0$. В зависимости от того, какое из чисел $b_{m} / b_{j}, b_{s} / b_{j}$ больше, имеем либо $1 \leqslant x \leqslant(k+d) /(k+1 / d)$, либо $(k+1 / d) /(k+d) \leqslant x \leqslant 1$. Таким образом, необходимо, чтобы $(k+d) /(k+1 / d) \leqslant d$ и $1 / d \leqslant(k+1 / d) /(k+d)$. Однако, оба этих неравенства равносильны и следуют из $d \geqslant 1$.

Предположим теперь, что $m=i$. По индуктивному предположению $\left|b_{i} / b_{j}\right| \leqslant 1 / a$, $b_{s} / b_{j}>0$. Если $b_{i} / b_{j} \geqslant b_{s} / b_{j}>0$, то $1 \leqslant x \leqslant(k+1 / a) /(k+1 / d) \leqslant d$, т.е. $k a+1 \leqslant a(k d+1)$. В противном случае $1 \geqslant x \geqslant(k-1 / a) /(k+d) \geqslant 1 / d$, т.е. $d(k a-1) \geqslant a(k+d)$.

Случай $s=i$ разбирается аналогично.

б) Пусть $l<0$. Тогда оценивать придется выражение

$$
y=\left|\frac{k l-b_{m} / b_{j}}{k l-b_{s} / b_{j}}\right|
$$

Как и выше, предположим сначала, что $m, s \neq i$. Дословно повторяя рассуждения части а), мы получим пару неравенств

$$
1 \leqslant y \leqslant \frac{k-1 / d}{k-d} \leqslant d \quad \text { и } \quad \frac{1}{d} \leqslant \frac{k-d}{k-1 / d} \leqslant y \leqslant 1 .
$$

Они сводятся к одному неравенству $k d-1 \leqslant d^{2}(k-d)$.

Если же $m=i$ (или $s=i$ ), то в случае $b_{i} / b_{j} \geqslant b_{s} / b_{j}>0$ получается неравенство $1 \geqslant y \geqslant(k-1 / a) /(k-1 / d) \geqslant 1 / d$. В противном случае $1 \leqslant y \leqslant(k+1 / a) /(k-d) \leqslant d$. Освобождаясь от дробей, получаем $a(k d-1) \leqslant d^{2}(k a-1)$ и $k a+1 \leqslant a d(k-d)$. 
Таким образом, для того чтобы был осуществим индуктивный переход, параметры $a, d$ должны удовлетворять системе неравенств:

$$
\begin{gathered}
\frac{k-\sqrt{k^{2}-4}}{2} \leqslant a \leqslant k-d, \quad 1 \leqslant d<k, \\
k a+1 \leqslant a(k d+1), \\
a(k+d) \leqslant d(k a-1), \\
k d-1 \leqslant d^{2}(k-d), \\
a(k d-1) \leqslant d^{2}(k a-1), \\
k a+1 \leqslant a d(k-d) .
\end{gathered}
$$

После этого достаточно заметить, что по предположению индукции $\left|b_{m} / b_{j}\right| \leqslant d<k$ и, в частности, все $b_{m}^{\prime}=b_{m}+k l b_{j}(m \neq j)$ того же знака, что и $k l b_{j}$.

Система (2)-(7), конечно, избыточна. Неравенство (7) сильнее неравенства (3), так как $d>0$. Оно также сильнее неравенства (5). Действительно, (7) можно переписать как $k+1 / a \leqslant d(k-d)$, а $(5)$ - как $d-1 / k \leqslant d(k-d)$. Точно так же можно показать, что (4) сильнее (6). В итоге система сокрашается до неравенств (2), (4), (7). Множество решений последней системы обозначим через $C(k)$. Несложно показать, что с уменьшением параметра $k$ множество $C(k)$ тоже “уменьшается". Иными словами, если $k_{1} \leqslant k_{2}$, то $C\left(k_{1}\right) \subseteq C\left(k_{2}\right)$. Действительно, функция $f(k)=\left(k-\sqrt{k^{2}-4}\right) / 2$ строго убьвает для всех $k>0$. Поэтому если $f\left(k_{1}\right) \leqslant a$, то тем более $f\left(k_{2}\right) \leqslant a$. Аналогично, если $a+d \leqslant k_{1}$ (или $d<k_{1}$ ), то заведомо $a+d \leqslant k_{2}$ (и $d<k_{2}$ ).

Положим $x=k_{2}-k_{1}$. Пусть, например, параметры $a, d$ удовлетворяют неравенству (4) с $k=k_{1}$. Чтобы получить (4) с $k=k_{2}$, достаточно добавить к левой части $a x$, а к правой $a d x$. Так как $a x \leqslant a d x$, неравенство останется верньм. Те же рассуждения проходят для (3).

В силу всего сказанного вьше достаточно показать, что $C(5) \neq \varnothing$. Это действительно так, например: пара $a=4 / 5, d=5 / 2$ лежит в $C(5)$. Теорема доказана.

ЗАмЕчАнИЕ. Конечно, число 5 не является наименьшим, при котором разрешима система неравенств (2)-(7). Однако, несложно проверить, что уже $C(4)=\varnothing$. В связи с этим было бы интересно понять, какова нижняя грань множества $\{k \mid C(k) \neq \varnothing\}$.

\section{СПИСОК ЦИТИРОВАННОЙ ЛИТЕРАТУРЫ}

[1] Зубков А.Н. Матричные представления свободных групп // 18-я Всесоюзная алгебраическая конференция. Тезисы сообщений. Ч. 2. Минск, 1983. С. 84.

[2] Каргаполов М.И., Мерзляков Ю. И. Основы теории групп. М.: Наука, 1977.

[3] Мерзляков Ю. И. Линейные группы // Итоги науки и техн. Алгебра. Топология. Геометрия. Т. 16. М.: ВИНИТИ, 1978. С. 35-89.

[4] Супруненко Д. А. Группы матриц. М.: Наука, 1972.

Омский государственный педагогический университет

E-mail : zubkov@iitam.omsk.net.ru

Поступило 31.07.97 